\title{
INTERESTING CASE OF COUGH FRACTURE IN AN ADULT
}

\author{
Praphull Deepankar1, Rakesh Roshan², Govind Kumar ${ }^{3}$, Amrita Pritam $^{4}$
}

${ }_{1}^{1}$ Assistant Professor, Department of General Medicine, IGIMS, Patna, Bihar, India.

2Senior Resident, Department of General Medicine, IGIMS, Patna, Bihar, India.

${ }_{3}^{3}$ Additional Professor, Department of General Medicine, IGIMS, Patna, Bihar, India.

4Junior Resident, Department of General Medicine, IGIMS, Patna, Bihar, India.

HOW TO CITE THIS ARTICLE: Deepankar P, Roshan R, Kumar G, et al. Interesting case of cough fracture in an adult. J. Evolution Med. Dent. Sci. 2018;7(22):2726-2727, DOI: 10.14260/jemds/2018/615

\section{PRESENTATION OF CASE}

A 32-year-old man, a known case of bronchial asthma was presented to our emergency department with chief complaint of sudden onset pain in the left lower chest wall after strong paroxysmal coughing due to aggravation of bronchial asthma symptoms. Chest pain was localised and aggravated with respiration without any radiation. There was no history of fever, abdominal pain, nausea and vomiting, diaphoresis or orthopnoea. History of any thoracic trauma, cardiac disease or osteoporosis due to chronic renal disease or any metabolic disease was not present. He was on intermittent oral and inhalational corticosteroid therapy, because of the uncontrolled bronchial asthma for last five years. On physical evaluation, he was tachypnoeic and vital was normal. He had tenderness over his left lower chest wall. Diffuse rhonchi were heard over both lung fields on auscultation.

Cough is generally self-limited and uncomplicated, but can be associated with complications, particularly when it is paroxysmal and violent. Rib fracture is among the uncommon complications of cough. In this article, we report a case of 32year-old male who presented with complaints of acute chest pain after paroxysmal coughing and was diagnosed with cough fracture of the ninth rib on subsequent workup. Rib fracture in otherwise healthy young individual after coughing is not common. The purpose of this case report is to call attention on rib fracture as infrequent and often an unrecognised cause of sudden chest pain, so that it should be considered as differential diagnosis of acute chest pain after common causes.

Coughing is a crucial defense mechanism in respiratory system and is usually uncomplicated, but sometimes can be with complications, especially when chronic. One of these complications is rib fracture. ${ }^{1}$ In this case report, we present a case of cough fracture in young patient.

\section{DIFFERENTIAL DIAGNOSIS}

On the Basis of Detailed History and Examinations, following are kept under Differential Diagnosis-

a. Primary Pleuritis.

b. Pleuropneumonia.

c. Musculoskeletal injury/abdominal muscle tear.

d. Rib fracture.

e. Acute coronary syndrome.

'Financial or Other Competing Interest': None.

Submission 08-04-2018, Peer Review 15-05-2018,

Acceptance 21-05-2018, Published 28-05-2018.

Corresponding Author:

Dr. Praphull Deepankar,

1A, Vrindavan Garden Apartment

Ashiana-Digha Main Road,

Patna-800025, Bihar, India

E-mail:drprafsjh@gmail.com

DOI: $10.14260 /$ jemds $/ 2018 / 615$

\section{CLINICAL DIAGNOSIS}

To confirm the diagnosis, patient was subjected to further investigation. Electrocardiogram showed normal axis, sinus tachycardia without ST changes. Arterial blood gas analysis did not show any significant abnormalities. Chest x-ray revealed fracture of the left ninth rib (Figure 1 and 2). Dualenergy x-ray absorptiometry was also performed, which excluded the possibility of osteopenia or osteoporosis. Other routine investigation was within normal limit. A clinical diagnosis of acute exacerbations of bronchial asthma and rib fracture was made.

\section{PATHOLOGICAL DISCUSSION}

The most common cause of rib fracture is trauma. Pathological fractures may be encountered secondary to malignancy, osteoporosis caused by older age, renal failure, pregnancy, chronic steroid use, mechanical ventilation and radiation therapy. ${ }^{2}$

Even though Hanak V et al reported bone densitometry were in the osteopenic or osteoporotic range in their study of cough stressed fracture, but cough induced rib fractures can occur in persons with normal bone density. ${ }^{1}$ In our case, bone density was also normal. In our case, the patient's risk factors included chronic coughing and chronic steroid use. These cough induced rib fractures are likely to be related to recurrent mechanical stress to the ribs caused by coughing. When the force of the cough is greater than the elastic limit of the ribs, the cough can cause a fracture over the vulnerable location. According to various studies, cough induced rib fractures occur most frequently on the lateral aspect of the fifth through ninth ribs. A second mechanism invokes an opposing force from the muscle attached to the same rib. This happens during violent coughing when the serratus anterior moves the ribs superiorly and laterally and the external oblique contracts to pull the ribs into medial alignment. Another mechanism of cough induced rib fractures says it is caused by a complex interplay between inspiratory and expiratory muscles. ${ }^{2}$ Cramer et al suggested that the upper five ribs are unlikely to get fractured from cough, because they are shorter and are supported posteriorly by the scapular musculature. As stated above, typical locations for rib fractures include the fifth through the ninth rib at the lateral aspect of the rib cage. ${ }^{3}$

The typical manifestations of rib fracture include localised pain in the chest wall, worsening discomfort during chest wall movement and tenderness over the chest. In many cases previously reported, the patient felt a snap on the side during a vigorous bout of coughing followed by a severe pain with signs of localised tenderness, crepitus and often even palpable bone fragments. 4 


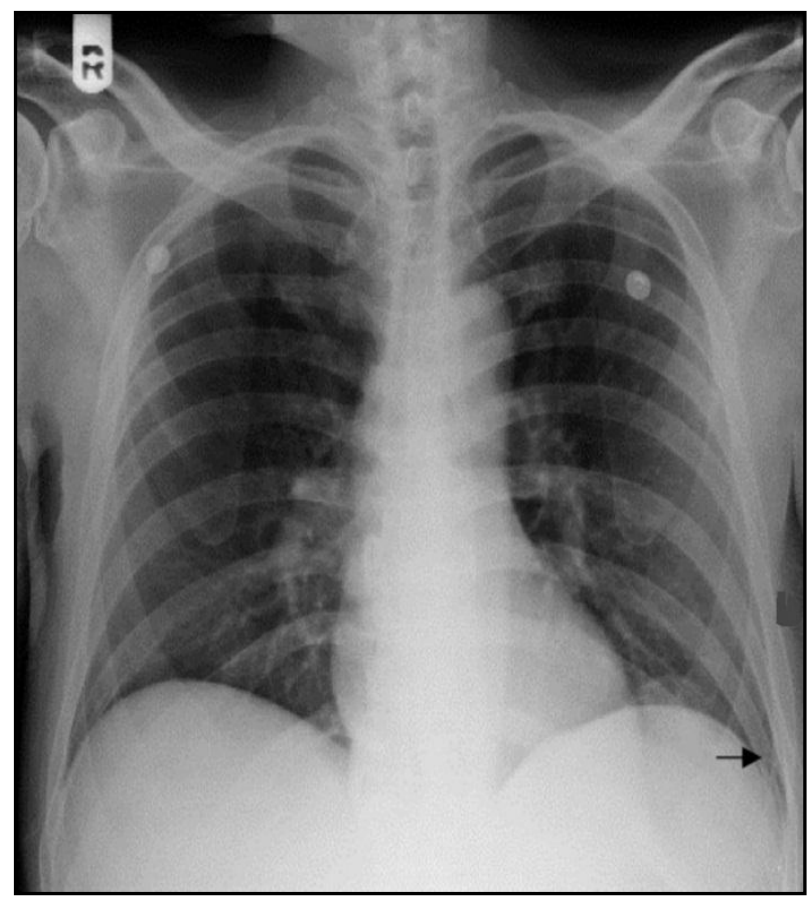

Figure 1. Plain Chest X-Ray showing Fracture of Rib

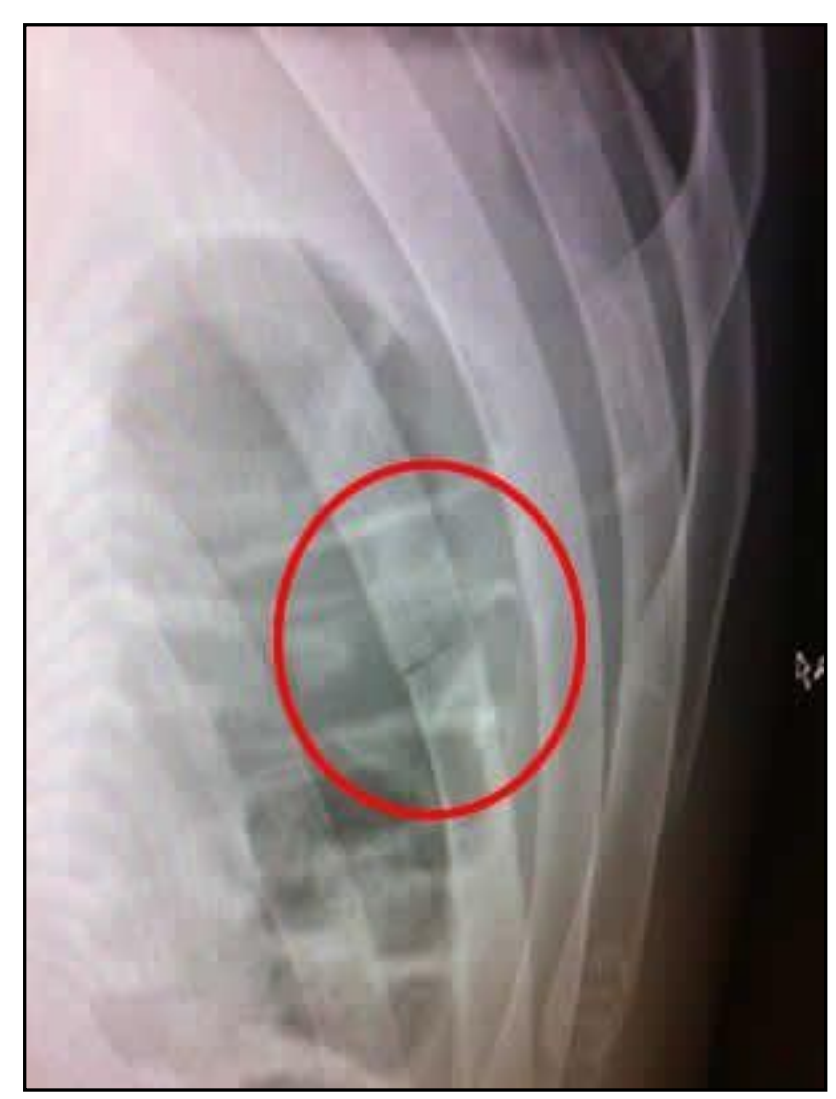

Figure. 2. Rib Fracture (Red Circle)

\section{DISCUSSION OF MANAGEMENT}

Chest roentgenograms are the most common and frequently used radiological procedures in the diagnosis of coughinduced fractures of the rib. But cough fracture are easily missed on standard x-ray views, because the lateral parts of the ribs are crowded and are apt to be obscured by scattered radiation, especially when soft films are taken which are designed to show lung lesions rather than bony defects. ${ }^{5}$ In addition computed tomography and radionuclide bone scan can use if chest roentgenograms is not conclusive. Cough fracture could be easily diagnosed, but abdominal muscle tears are frequently missed. Hence, computed tomography seems to be essential for an accurate diagnosis if needed. 6

The treatment of spontaneous rib fractures consists of pharmacological treatment and close clinical observation. If complications such as pneumothorax, pleural effusion, subcutaneous emphysema, haemothorax, haematoma, diaphragmatic rupture or lung hernia are observed, tube thoracostomy and surgical intervention may be indicated.

\section{FINAL DIAGNOSIS}

Cough-induced non-complicated rib fracture with bronchial asthma without evidence of osteoporosis was our final diagnosis. The patient was put on conservative management of acute asthmatic attack and rib fracture. Patient was discharged after symptomatic improvement. There were no complications during follow-up.

It is not common to have rib fracture in otherwise healthy young man due to persistent coughing. So, in patients that present with persistent coughing and sudden chest pain, cough-induced rib fractures should be considered in the differential diagnosis of chest pain. Early diagnosis and treatment may prevent the complications.

\section{REFERENCES}

[1] Hanak V, Hartman TE, Ryu JH. Cough-induced fib fractures. Mayo Clinic Proceedings 2005;80(7):87982.

[2] Kilic D, Findikcioglu A, Hatipoglu A. Spontaneous rib fracture caused by coughing: report of two cases. Turkey Klinikleri J Med Sci 2007;27(3):468-70.

[3] Aysel S, Abdussamet B. Cough-induced rib fracture: a case report. Eastern Journal of Medicine 2015;20:2545.

[4] Kawahara H, Baba H, Wada M. Multiple rib fractures associated with severe coughing-a case report. Int Orthop 1997;21(4):279-81.

[5] Narayanasamy SN, Selvaraju S, Gopalakrishnan N, et al. Cough fracture: stress on the ribs. Int J Res Med Sci 2015;3(3):808-9.

[6] De Maeseneer M, De Mey J, Debaere C, et al. Rib fractures induced by coughing: an unusual cause of acute chest pain. Am J Emerg Med 2000;18(2):194-7. 